\title{
REGRESSION MODELLING OF THE INTERACTION BETWEEN THE SOLAR WIND AND THE TERRESTRIAL MAGNETOSPHERE
}

\author{
A. S. Parnowski, A. Yu. Polonska \\ Space Research Institute of NASU \& NSAU, \\ prosp. Glushkova, 40, build. 4/1, Kyiv, UA-03680, Ukraine, \\ e-mail: parnowski@ikd.kiev.ua \\ (Received November 27, 2011)
}

\begin{abstract}
On the basis of the regression modelling method we investigated temporal variations of $D_{\mathrm{ST}}$, $a_{\mathrm{P}}$ and $K_{\mathrm{P}}$ indices. The developed models also provide some new information about the underlying physics of the interaction between the solar wind and the terrestrial magnetosphere.
\end{abstract}

Key words: space weather, solar wind, magnetosphere, regression modelling.

PACS number(s): 02.70.Rr, 94.05.Sd, 94.05.sx, 94.30.Lr, 94.30.vf

\section{INTRODUCTION}

Today our understanding of the interaction between the solar wind and the terrestrial magnetosphere is not deep enough to create physics-based predictive models. For this reason, different approaches should be tried. Today the optimal combination of virtues and vices is provided by the methods involving time series analysis and data mining [1]. They provide a moderate prediction time (up to several hours) with the highest available accuracy ( $>80 \%$ ). They are very effective and easy to use, but strongly depend on satellite data availability. These are "black box" or "input-output" models, which seek only to reproduce the system's output in response to changes of its inputs. The model terms are usually physically interpretable and thus useful for constructing new phenomenological models. For this reason, this method can not only provide a space weather forecast per se, but also improve our knowledge of the underlying physics and thus increase the efficiency of other methods. Most of these methods have a feature in common: they lead to a regression relation at some point, so it seems natural to skip all the preliminary steps and instantly use the regression analysis without the unnecessary multiplication of entities.

In our article [2] we described a new approach, named "regression modelling". This approach can provide accurate short-term and, to a certain extent, medium-term forecasts of geomagnetic indices. It gives new information about the underlying physics, thus contributing to the solar-terrestrial physics.

In this article we shall discuss the physical implications of the regression modelling method. Some preliminary descriptions of different aspects of this method can also be found in the articles [3-6]. For the description of the method itself we refer to the articles $[2,7]$.

We will only mention here that we consider a discrete dynamical system (in our case, the terrestrial magnetosphere) with an unknown number $K_{\text {tot }}$ of inputs $u_{k}$ and one output $y$ (one of the geomagnetic indices). At each step $t$ we know only $K<K_{\text {tot }}$ inputs $u_{k}(t), k=\overline{1, K}$ $(\overline{1, K}$ means all the integer numbers from 1 to $K$ inclu- sively) and the output $y(t)$. Then at an arbitrary step $T$ we can write the system's output in the form

$$
y(T+\Theta)=y^{*}(T+\Theta)+\Delta y(T+\Theta),
$$

where $\Theta$ is the lead time of the forecast (the number of hours the forecasted value is ahead of the last measured value), $y^{*}(T+\Theta)$ is the predicted value, and $\Delta y(T+\Theta)$ is the uncertainty, which we assume to be stochastic. We are also forced to assume that all the values are distributed normally to be able to apply the methods of mathematical statistics, though this is, of course, not always true. We also assume that the statistical properties of the dynamical system do not change on the time scale $\Theta$. The predicted value $y^{*}(T+\Theta)$ is expressed through a partial regression relation [8]:

$$
y^{*}(T+\Theta)=\sum_{i=1}^{m} C_{i} x_{i}(T),
$$

where $x_{i}, i=\overline{1, m}$ are the regressors, which are arbitrary functions of input quantities $u_{k}(t)$, which are already measured at the time $T$ when the forecast is made, $C_{i}, i=\overline{0, m}$ are the regression coefficients, and $m$ is the number of variable regressors.

We choose the regressors $x_{i}$ in the form of products of powers of the input quantities

$$
x_{i}(t)=\prod_{k=1}^{K} u_{k}^{p_{k}}(t-l), l=\overline{0, L},
$$

where $p_{k}$ are powers, which can be equal to zero or any natural number, $l$ is the lag, and $L$ is the maximal lag. In contrast to empirical models we do not add fitting parameters and all the regressors have physical meaning. Note that different sets of regressors should be taken for different values of the lead time $\Theta$.

The coefficients $C_{i}$ are determined by the generalised least squares method over a large sample of solar wind and geomagnetic data, with equal statistical weights of all points. The statistical significances of the regressors are determined according to Fisher's F-test $[8,9]$. 


\section{DESCRIPTION OF DATA USED}

As before, we used the OMNI 2 database [10], which contains IMF, solar wind and geomagnetic data, averaged over 1-hour intervals (at the time of publication it contained 54 parameters, starting from 1 January 1963). This database covers a vast number of spacecraft. In recent years the data come from the ACE spacecraft located in the first Lagrange (L1) point, also called a libration point, which is situated along the Earth-Sun axis approximately $0.01 \mathrm{AU}$ (1.5 millions of kilometres) from the Earth. For typical interplanetary conditions $\left(V=470 \mathrm{~km} \mathrm{~s}^{-1}\right)$ a spacecraft located there provides real-time data with approximately 40-minute lead time.

The geomagnetic data come from Kyoto WDC for Geomagnetism $\left(D_{\mathrm{ST}}, \mathrm{AE}\right)$ and from Geoforschung Zentrum Potsdam ( $K_{\mathrm{P}}, a_{\mathrm{P}}$ and associated indices). Since September 2011 the final $D_{\mathrm{ST}}$ index is available up to $31 \mathrm{De}-$ cember 2008. The most recent definitive $K_{\mathrm{P}}$ index is published twice per month.

The data before 1976 are scarce and of poor quality and their inclusion in the dataset negatively impacts its characteristics. Also, 1976 was the first year of the solar cycle 21. For these reasons we used a training sample that ranges from 1 January 1976 to 31 December 2000, thus forming a continuous 25-year time series with the total of $n=219168$ datapoints. For the $D_{\mathrm{ST}}$ index the mean is $\overline{D_{\mathrm{ST}}}=-18.3 \mathrm{nT}$, the median is $-23 \mathrm{nT}$, the mode is $8 \mathrm{nT}$, and the standard deviation is $\sigma_{D_{\mathrm{ST}}}=24.6 \mathrm{nT}$. The distribution of the $D_{\mathrm{ST}}$ index visually represents a normal one, but the Pearson's $\chi^{2}$ test [8] disproves this null-hypothesis at the $99.99 \%$ confidence level $\left(\chi^{2}=416125.8\right)$. This is due to flatter wings of the distribution, which are caused by the periodicities of the ACF. For the $a_{\mathrm{P}}$ index the mean is $\overline{a_{\mathrm{P}}}=14.9 \mathrm{nT}$, the median is $5 \mathrm{nT}$, the mode is $27 \mathrm{nT}$, and the standard deviation is $\sigma_{a_{\mathrm{P}}}=20.0 \mathrm{nT}$.

Unfortunately, during intense storms the instruments aboard the spacecraft are often turned off to prevent permanent damage to them and some or all of the input values are unavailable. By rejecting filled values from the time series, we obtain the sample, which can be divided into different subsamples for specific purposes. Of course, the resulting sample will vary according to the exact dependences of the regressors on the input quantities. For example, if a model contains a regressor, which depends on the ion density with a lag of 5 hours, then we have to reject each datapoint whose 5 th predecessor contained a filled value of the ion density.

\section{MEMORY OF THE GEOMAGNETIC INDICES}

Let us begin with investigating temporal variations of the geomagnetic indices. It seems natural to do so by analyzing the autocorrelation functions (ACF) for the $D_{\mathrm{ST}}$ (Figure 1) and the $a_{\mathrm{P}}$ indices (Figure 2). A brief glance at the ACF is enough to tell that neither of the geomagnetic indices can be treated as a Markov process.
In fact, both the $D_{\mathrm{ST}}$ and the $a_{\mathrm{P}}$ indices are periodically correlated.

One can see that in both cases the ACF tends to a sinusoid with a period close to half a year. Besides the half-ayear periodicity one can also notice the 27 -day periodicity, caused by Carrington rotation of the Sun. The former is caused by seasonal variations, which yields a question: if there were no temporal variations, what would ACF tend to at large offsets? If the distribution of $D_{\mathrm{ST}}$ and $a_{\mathrm{P}}$ was normal, the answer would be zero. However, the distribution slightly deviates from the normal one (the distribution has flatter wings), so the ACF can tend to some non-zero quantity.

To determine this quantity we need to remove temporal variations. For this purpose we need to calculate the $\mathrm{ACF}$ of a random sample with the same statistical characteristics as the actual sample. The easiest way to get such a sample is to process the actual sample with a permutation method, which is widely used in astronomy e.g. for the calculation of 2-point correlation functions. This method involves a random shuffling of the sample. Using this method many times (10 000 times in our case) and calculating the correlation coefficient each time, we get the distribution of the correlation coefficient in the Monte Carlo framework.

The distribution of the coefficient for this sample appeared to be very close to a normal distribution. For the $D_{\mathrm{ST}}$ index the mean was equal to $0.008 \mathrm{nT}$ and the variance - to $5.1 \cdot 10^{-6} \mathrm{nT}^{2}$. The maximum recorded value in 10000 trials was equal to $0.015 \mathrm{nT}$ for both indices. The top and the mean values are depicted on Figures 1 and 2 by horizontal lines. As one can see, in reality the correlation coefficient exceeds this value at most times due to temporal variations. The ACF of the $D_{\mathrm{ST}}$ index crosses the top line for the first time at about 6000 hours, though the difference between the ACF and the sine with a half-year period crosses it at about 2000 hours, which is about 3.5 27-day Carrington periods. The ACF of the $a_{\mathrm{P}}$ index crosses the top line at about 1200 hours, though the difference between the ACF and the sine with 27-day period crosses it at about 1000 hours. We will take the latter value as a rough estimation of $L$. This hints that rather old values of the geomagnetic indices can be quite significant. A similar situation was reported by Johnson and Wing [11] regarding $K_{\mathrm{P}}$ : "the significance is often quite large for extended periods of time (10-20 days)".

To investigate this effect, we should construct an autoregression model [6]

$$
y_{\mathrm{AR}}^{*}(T+\Theta)=C_{0}+\sum_{l=0}^{L} C_{l} y(T-l) \text {. }
$$

Applying the F-test we can determine which previous $D_{\mathrm{ST}}$ and $a_{\mathrm{P}}$ values are statistically significant. We did not search statistically significant values for $L \geqslant 1000$, but it is possible that there are even older statistically significant values. In fact, the final models still contained statistically significant values of $D_{\mathrm{ST}}$ as far as 900 hours ago, which is about 37.5 days (see Figure 3 ). The statistical significance of these oldest values can reach $99.95 \%$. 


\section{TEMPORAL VARIATIONS OF GEOMAGNETIC INDICES}

On Figure 1 one can see a clear seasonal dependence of the $D_{\mathrm{ST}}$ index. Indeed, if we select two subsamples, corresponding to the summer and the winter in the northern hemisphere, bounded by vernal and autumnal equinoxes, and verify the hypothesis that the difference between the corresponding average $D_{\mathrm{ST}}$ values is statistically significant using a one-sided Student's test [8], we obtain $t_{\infty}=80.3$, which is well over $99.95 \%$ significant. The values of $t_{\infty}$ corresponding to $99 \%$ and $99.95 \%$ confidence levels are equal to 2.3 and 3.3 , respectively. For the diurnal asymmetry the Student's test gives $t_{\infty}=8.8$, which corresponds to the significance level of $99.95 \%$. Note that formally the Student's test is applicable only to normally distributed values and the distribution of $D_{\mathrm{ST}}$ has flatter wings than the normal one. However, taking into account the obtained large values of $t_{\infty}$, we can be sure of the qualitative conclusions made.

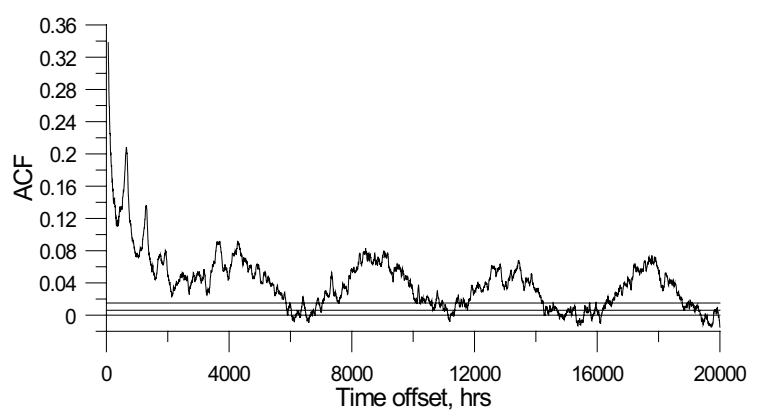

Fig. 1. Autocorrelation function of the $D_{\mathrm{ST}}$ index.

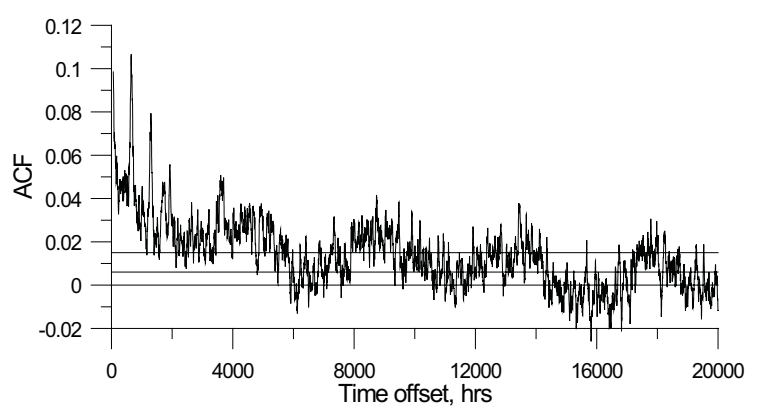

Fig. 2. Autocorrelation function of the $a_{\mathrm{P}}$ index.

This dependence was described in many articles, e.g. [12-15], but the reason behind it is still disputed. Most authors believe these asymmetries are caused by either of two cusps turning to the sunlit side due to the annual rotation of the Earth with respect to the Sun. However, O'Brien and McPherron [15] state that this mechanism can be held responsible only for $17 \%$ of the observed asymmetry. Takalo and Mursula [14] connected the diurnal variations of $D_{\mathrm{ST}}$ with an inhomogeneous distribution of $D_{\mathrm{ST}}$ network stations with respect to the longitude.

In our opinion, this behaviour is most likely caused by an asymmetry of the $D_{\mathrm{ST}}$ stations with respect to the geomagnetic equator. In fact, only the Hermanus station is located in the southern hemisphere (dipole latitude $\left.-33.3^{\circ}\right)$, while the other 3 stations are located in the northern hemisphere (Kakioka $+26.0^{\circ}$, Honolulu $+21.1^{\circ}$, San Juan $+28.0^{\circ}$ ).

The official definition of the $D_{\mathrm{ST}}$ index is [16]

$$
D_{\mathrm{ST}}(t)=\frac{\left\langle\Delta H(t)-S_{q}(t)\right\rangle}{\langle\cos \theta\rangle},
$$

where $\Delta H(t)$ is the difference between the observed and the baseline values of the $\mathrm{H}$-component of the geomagnetic field,

$$
S_{q}(t, s)=\sum_{m} \sum_{n} A_{m n} \cos \left(m t+\alpha_{m}\right) \cos \left(n s+\beta_{n}\right),
$$

is the solar quiet daily variation, $s$ is the current month, $\theta$ is the geomagnetic latitude, and $\langle\bullet\rangle$ is an average over 4 contributing stations. Since it does not depend on the sign of $\theta$, any sources in the northern hemisphere will affect the $D_{\mathrm{ST}}$ index 3 times stronger than their southern counterparts. Note that temporal variations of the $D_{\mathrm{ST}}$ index are provided not only by actual temporal variations of the H-component, but also by the term (6), which explicitly contains them.

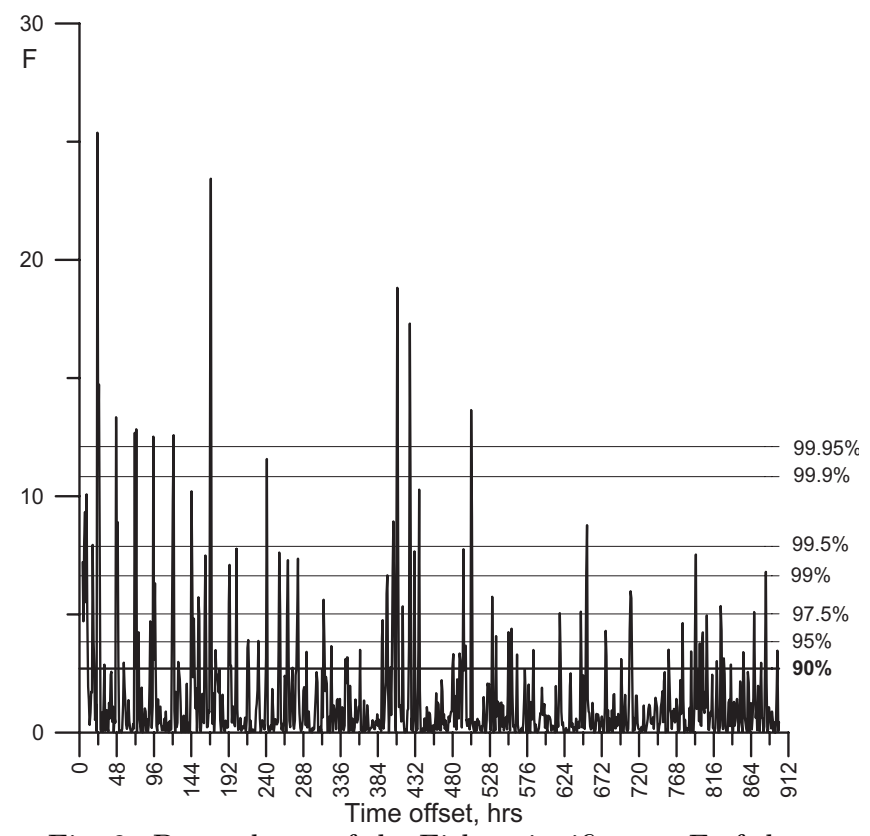

Fig. 3. Dependence of the Fisher significance $F$ of the previous $D_{\mathrm{ST}}$ value on its lag for the autocorrelation model with 1 hour lead time.

Usually, the $D_{\mathrm{ST}}$ index is associated with the ring current, which is highly asymmetric during the geomagnetic storm [17-19]. However, the $\Delta H(t)$ term includes all the sources of the magnetic field, such as ionospheric currents, power lines, industrial facilities, railroads and so on [20]. This fact explains, among others, the 7-day periodicity, which is of purely anthropogenic origin. Also, initially the $D_{\mathrm{ST}}$ index is derived from very noisy data, and it is possible that additional errors were introduced during its processing. 
Taking this known geoeffective factor as an example we demonstrate how easily one can take it into account using the regression approach. To do so one should simply add the synthetic inputs

$$
u_{K+1}(t)=\sin ((\operatorname{DOY}(t)-80) \pi / 182.62)
$$

and

$$
u_{K+2}(t)=\cos ((\operatorname{DOY}(t)-80) \pi / 182.62)
$$

Here DOY is the day of the year, 80 is the number of days between January 1 and the vernal equinox, and 182.62 is the number of days in half a year. The first of these terms is significant and describes the summer-winter asymmetry, and the second one (which appears statistically insignificant) describes an absent spring-autumn asymmetry. Likewise, for the diurnal asymmetry the corresponding synthetic inputs will be

$$
u_{K+3}(t)=\sin ((\mathrm{UT}(t)-2) \pi / 12)
$$

and

$$
u_{K+4}(t)=\cos ((\mathrm{UT}(t)-2) \pi / 12)
$$

Here 2 is the time difference between UT and the local time at the northern magnetic pole, and 12 is the number of hours in half a day. Both these terms are significant.

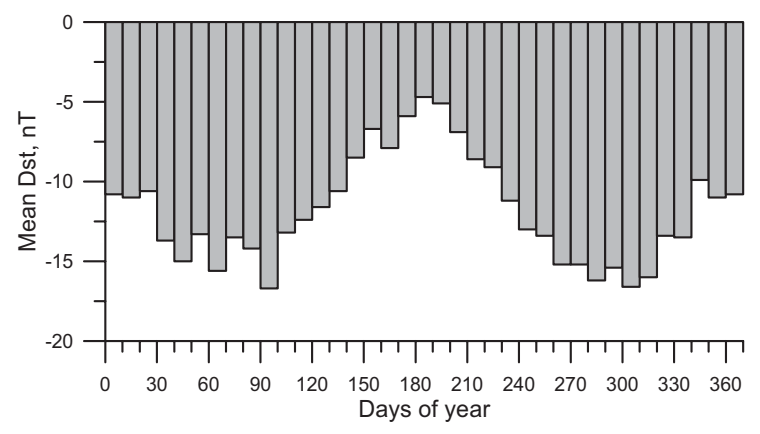

Fig. 4. Seasonal variation of the $D_{\mathrm{ST}}$ index.

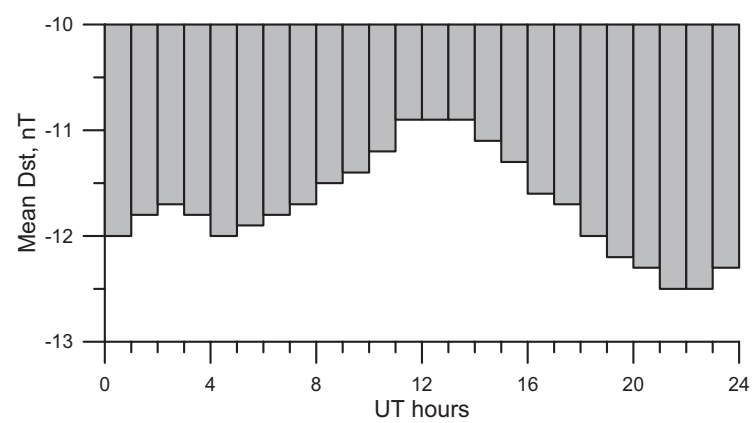

Fig. 5. Diurnal variation of the $D_{\mathrm{ST}}$ index.

The coefficient of the regressor, equal to $u_{K+3}(t)$, is less than the actual difference between the summer and the winter mean $D_{\mathrm{ST}}$ values by an order of magnitude. This can be explained in the following way: there are other regressors, which depend on the parameters with statistically significant summer-winter asymmetry, e.g. previous $D_{\mathrm{ST}}$ values. They provide the lion share of the summer-winter asymmetry of the $D_{\mathrm{ST}}$ index. A good example of such a parameter is the international sunspot number $R$, which has a 27-day periodicity due to Carrington's rotation of the Sun. Nevertheless, there is a small difference which cannot be expressed with these terms. Including it into regression, we obtain these statistically significant regressors.

There is another possible explanation of this effect. Let us consider as an example a value

$$
X(t)=\text { const }+A \sin \omega t .
$$

In the regression it will look like

$$
\begin{aligned}
X(t+\Delta t) & =X(t)+A(\sin \omega(t+\Delta t)-\sin \omega t) \\
& =X(t)+A((\cos \omega \Delta t-1) \sin \omega t \\
& +\cos \omega t \sin \omega \Delta t) .
\end{aligned}
$$

The first term in brackets is of the order $(\omega \Delta t)^{2}$, and the second one is of the order $(\omega \Delta t)$ in the natural assumption that $\omega \Delta t \ll 1$. So, it will seem that the coefficient is $A(\omega \Delta t)$ rather than $A$. Note that this is just an example and has nothing to do with the actual regressors.

However, the distribution of mean $D_{\mathrm{ST}}$ values vs. the day of the year (Figure 4) is much more complicated. Among its features there is a strong asymmetry between the summer and the winter on one side and the spring and the autumn on the other. To take it into account we introduced additional terms into our regression, which are the powers of $u_{K+1}(t)$ and their products with the powers of $u_{K+2}(t)$. The sum of regressors with the corresponding coefficients is very similar to the actual distribution (Figure 5). Note that the coefficients were obtained independently from the distribution.

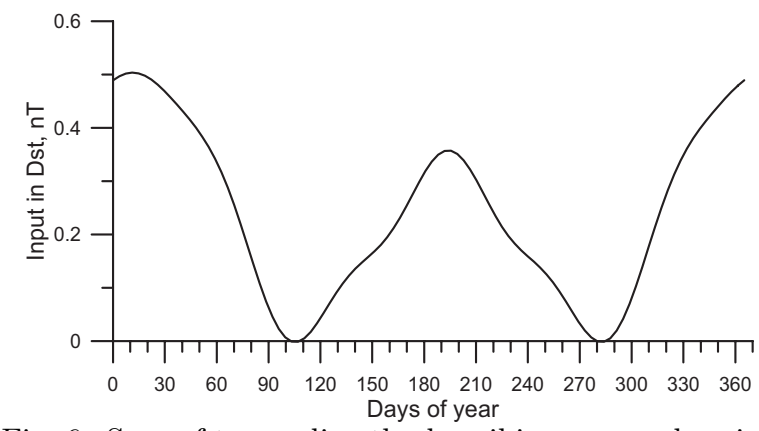

Fig. 6. Sum of terms directly describing seasonal variation of the $D_{\mathrm{ST}}$ index.

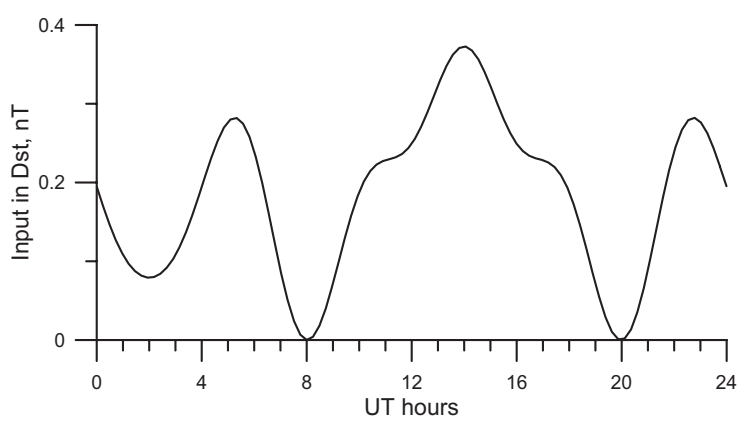

Fig. 7. Sum of terms directly describing diurnal variation of the $D_{\mathrm{ST}}$ index. 
We did the same thing with the diurnal asymmetry (Figures 6,7$)$. The cross-product $u_{K+1}(t) \cdot u_{K+3}(t)$ is also significant and should be included in the regression. After this we obtained a joint distribution of seasonal and diurnal variations of the $D_{\mathrm{ST}}$ index, which contains 18 regressors (Figure 8). Increasing the number of the regressors describing temporal variations of the geomagnetic activity we can improve the accuracy of this distribution. In particular, one could add 11-year Schwabe's and 22-year Hale's solar cycles, higher powers of $u_{K+1}(t), \ldots, u_{K+4}(t)$, etc.

\section{NEW GEOEFFECTIVE PARAMETERS}

Now let us discuss the parameters whose geoeffectiveness was previously unknown: the latitudinal and the longitudinal bulk flow angles of the solar wind and demonstrate that they are indeed geoeffective.

The distribution of the latitudinal flow angle $\theta_{V}$ and the corresponding mean $D_{\mathrm{ST}}$ value is plotted on Figure 9. The distribution looks similar to a normal distribution with $\sigma_{\theta_{V}}=2.97^{\circ}$ and $\overline{\theta_{V}}=0.64^{\circ}$, and this nullhypothesis is supported by Pearson's $\chi^{2}$ test at more than $99.99 \%$ confidence level ( $\left.\chi^{2}=3284.9, n=118198\right)$. However, the number of points with deviations exceeding $3 \sigma$ is larger than it follows from the normal distribution (787 versus 319 ). The minimal and maximal values of $\theta_{V}$ are equal to $-32.0^{\circ}$ and $29.7^{\circ}$, respectively.

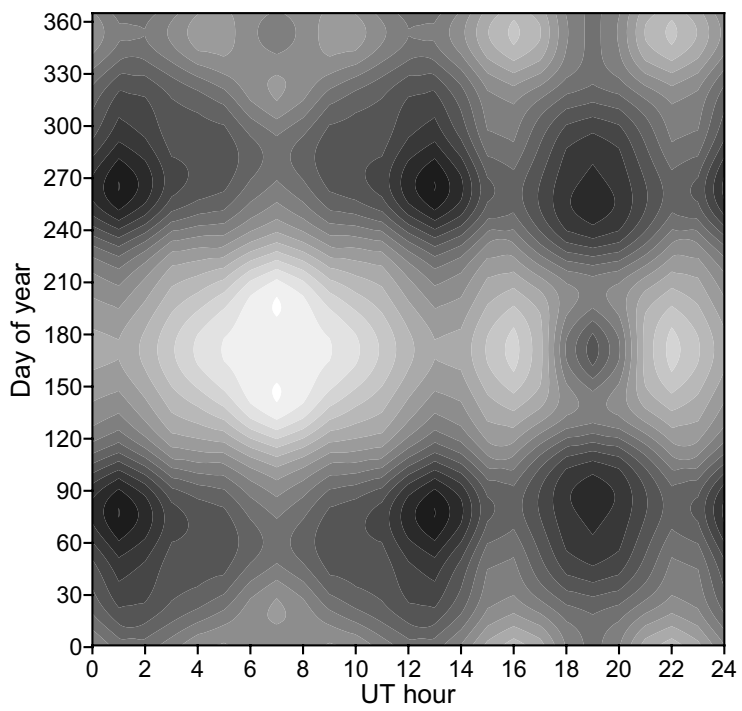

Fig. 8. Temporal variation of the $D_{\mathrm{ST}}$ index. Darker spots correspond to lower values.

If we ignore the wing bins in the distribution of mean $D_{\mathrm{ST}}$ values against $\theta_{V}$, which are somewhat random due to a small amount of points in them, we will notice a slight almost linear trend. If we plot the sum of terms containing $\theta_{V}$ (Figure 10), we will notice a similar trend. If we select two subsamples, one $-4^{\circ}<\theta_{V}<0^{\circ}$ and the other $0^{\circ}<\theta_{V}<4^{\circ}$, and verify the hypothesis that the difference between the corresponding average $D_{\mathrm{ST}}$ values is statistically insignificant using a one-sided Stu- dent's test, we obtain $t_{\infty}=5.9$, which is over $99.95 \%$ significant.

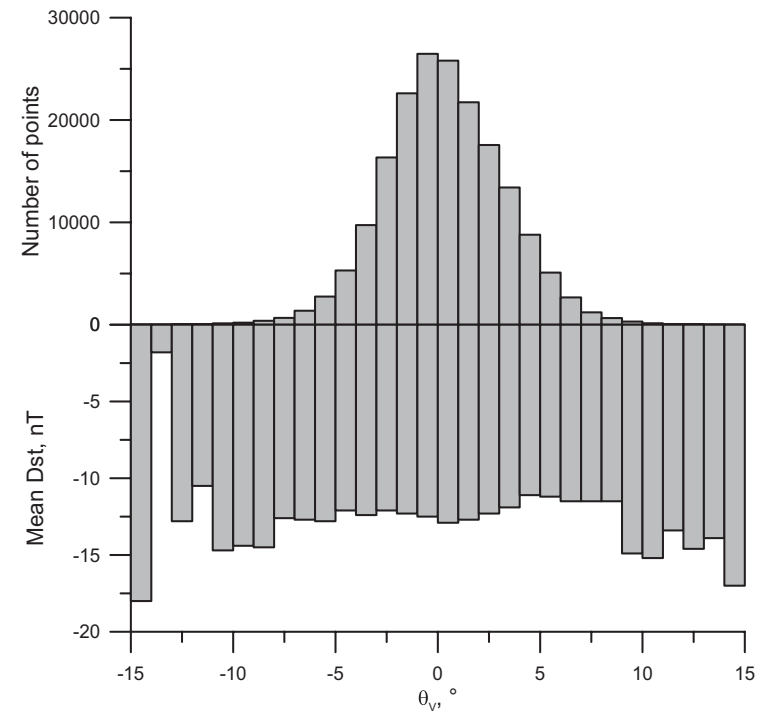

Fig. 9. Distribution of the latitudinal flow angle and the corresponding mean $D_{\mathrm{ST}}$ values.

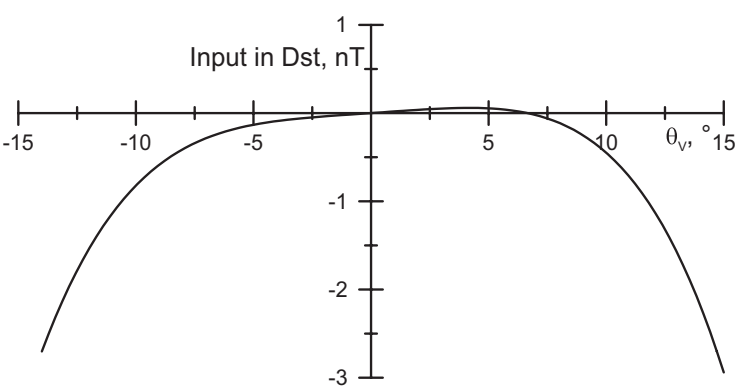

Fig. 10. Sum of terms describing the latitudinal flow angle.

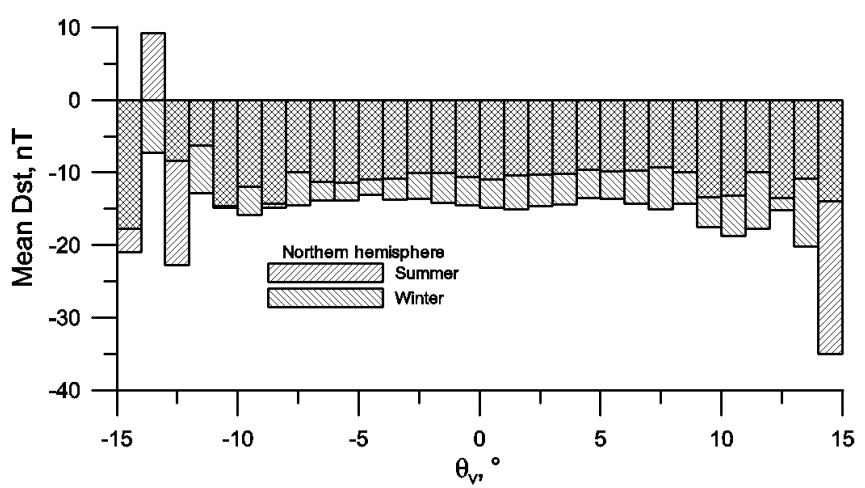

Fig. 11. Seasonal dependence of the latitudinal flow angle's input in the $D_{\mathrm{ST}}$ index.

If we divide the sample in two subsamples, one for northern summer and one for northern winter, and plot the corresponding histograms on Figure 11, we will see that the summer distribution has an obvious linear trend, but the winter one has not. If we apply the Student test to the same intervals now, we obtain that $t_{\infty}=5.44$ in the summer and $t_{\infty}=0.059$ in the winter. The former corresponds to more than $99.95 \%$ significance, while the 
latter - to less than $10 \%$. This proves that seasonal variations of the geomagnetic indices are at least partially caused by the actual physical processes.

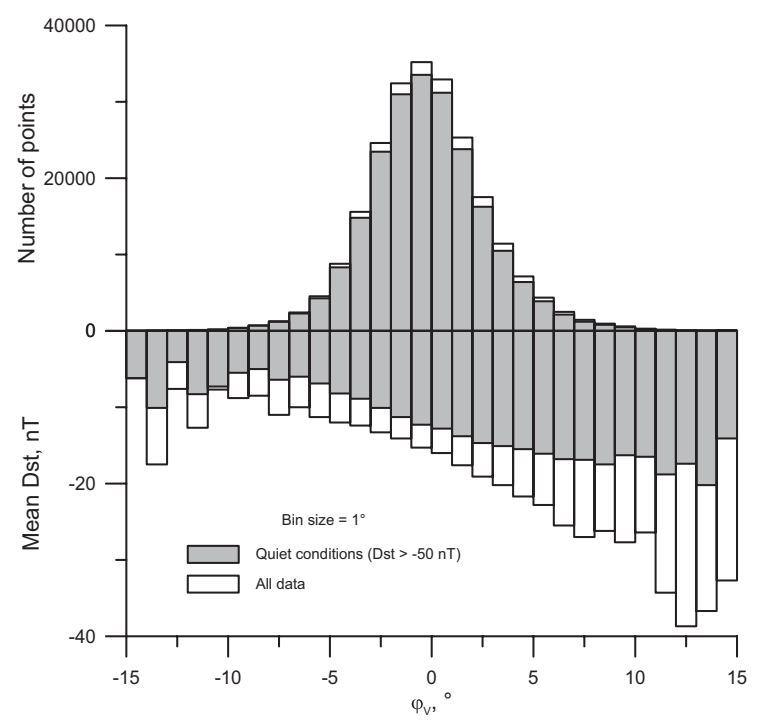

Fig. 12. Distribution of the longitudinal flow angle and the corresponding mean $D_{\mathrm{ST}}$ values. Grey columns correspond to quiet conditions, white columns - to all data.

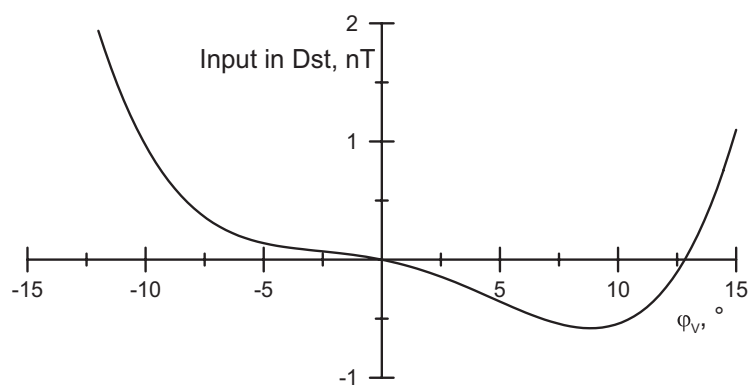

Fig. 13. Sum of terms describing the longitudinal flow angle.

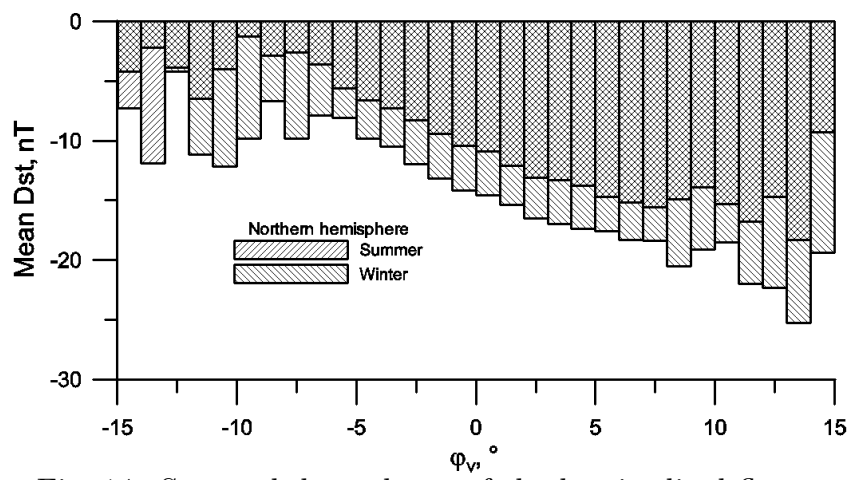

Fig. 14. Seasonal dependence of the longitudinal flow angle's input in the $D_{\mathrm{ST}}$ index.

The longitudinal flow angle $\varphi_{V}$ appeared to be even more significant than the latitudinal flow angle. Its distribution together with the corresponding mean $D_{\mathrm{ST}}$ values is plotted on Figure 12, where white bars show the complete sample, and the grey bars show the quiettime sample with $D_{\mathrm{ST}}>-50 \mathrm{nT}$. Like the latitudinal flow angle, the distribution of the longitudinal flow angle resembles a normal distribution with $\sigma_{\varphi_{V}}=2.9^{\circ}$ and $\overline{\varphi_{V}}=-0.1^{\circ}$. The Pearson's $\chi^{2}$ test supports the relevant null-hypothesis at more than $99.99 \%$ confidence level $\left(\chi^{2}=7871.0, n=129236\right)$. However, the number of points with deviations exceeding $3 \sigma$ is still larger than it follows from the normal distribution (1213 versus 349). The minimal and maximal values of $\varphi_{V}$ are equal, respectively, to $-20.7^{\circ}$ and $19.0^{\circ}$.

A significant trend is the most prominent feature of this figure. If we plot a sum of regressors, which contain $\varphi_{V}$ (Figure 13), we shall see a very similar trend. If we select two subsamples, one $-8^{\circ}<\varphi_{V}<-4^{\circ}$ and other $4^{\circ}<\varphi_{V}<8^{\circ}$, and verify the hypothesis that the difference between the corresponding average $D_{\mathrm{ST}}$ values is statistically insignificant using a one-sided Student's test, we obtain $t_{\infty}=37.1$, so the null-hypothesis should be rejected at well over $99.95 \%$ confidence level.

Like before, we separately plotted the distributions for summer and winter subsamples (Figure 14). We see that the trend is identical on both plots, so the corresponding effect is season-independent.

\section{CONCLUSION}

We demonstrated how easily one can take into account temporal variations in this method's framework. Note that these regressors do not depend on space-borne parameters and can be used alongside the previous values of geomagnetic indices. In this case they improve the forecast skill of the model and make some of the autoregression terms insignificant.

Also, we demonstrated that our method is truly capable of pointing out new geoeffective parameters and verified the geoffectiveness of two such values.

\section{ACKNOWLEDGEMENTS}

The OMNI data were obtained from the GSFC/SPDF OMNIWeb interface at http://omniweb.gsfc.nasa.gov/

We acknowledge the use of the geomagnetic data from Kyoto WDC for Geomagnetism (http://wdc.kugi. kyoto-u.ac.jp/) and Geoforschung Zentrum Potsdam (http://www-app3.gfz-potsdam.de/kp_index/).

The research leading to these results has received funding from the European Commission's Seventh Framework Programme (FP7/2007-2013) under the grant agreement No 263506 (AFFECTS project, www.affectsfp7.eu).

This research was supported by the Grant of the President of Ukraine for Young Scientists No GP/F32/0020.

This research was supported by the Grant of the National Academy of Sciences of Ukraine for Young Scientists. 
[1] A. S. Parnowski et al., Space Sci. Technol. 16, 90 (2010).

[2] A. S. Parnowski, J. Phys. Stud. 15, 2002 (2011).

[3] A. S. Parnowski, Space Sci. Technol. 14, 48 (2008).

[4] A. S. Parnowski, J. Phys. Stud. 13, 4003 (2008).

[5] A. S. Parnowski, Astrophys. Space Sci. 323, 169 (2009).

[6] A. S. Parnowski, Earth Planets Space 61, 621 (2009).

[7] A. S. Parnowski, J. Autom. Inform. Sci. 41, 128 (2009).

[8] D. J. Hudson, Statistics Lectures on Elementary Statistics and Probability (Geneva, CERN, 1964).

[9] R. A. Fisher, Statistical methods for research workers (London, Oliver and Boyd, 1954).

[10] J. H. King, N. E. Papitashvili, J. Geophys. Res. 110, A02104 (2005).

[11] J. R. Johnson, S. Wing, U.S. DoE Report PPPL3919rev, http://www.pppl.gov/pub_report/2004/ PPPL-3919rev.pdf (2004).
[12] E. W. Cliver et al., J. Geophys. Res. 105(A2), 2413 (2000).

[13] W. Lyatsky et al., Geophys. Res. Lett. 28, 2353 (2001).

[14] J. Takalo, K. Mursula, J. Geophys. Res. 106(A6), 10905 (2001).

[15] T. P. O'Brien, R. L. McPherron, J. Geophys. Res. 107(A11), 1341 (2002).

[16] M. Sugiura et al., IAGA Bull., 40 (1991); http:// wdc.kugi.kyoto-u.ac.jp/dstdir/dst2/onDstindex.html

[17] E. E. Antonova et al., Annales Geophysicae 27, 4069 (2009).

[18] E. E. Antonova et al., Adv. Space Res. 43, 628 (2009).

[19] A. Y. Ukhorskiy et al., J. Geophys. Res. 111, A11S03 (2006).

[20] W. H. Campbell, J. Atm. Terr. Phys. 58, 1171 (1996).

\title{
РЕГРЕСІЙНЕ МОДЕЛЮВАННЯ ВЗАЄМОДІЇ СОНЯЧНОГО ВІТРУ 3 МАГНІТОСФЕРОЮ ЗЕМЛІ
}

\author{
О. С. Парновський, А. Ю. Полонська \\ Інститут космічних дослідженъ НАНУ та НКАУ \\ просп. Глушкова, 40, корп. 4/1, Київ, 03680, Украӥна
}

На основі методу регресійного моделювання ми дослідили часові варіації $D_{\mathrm{ST}}, a_{\mathrm{P}}$ та $K_{\mathrm{P}}$-індексів. Розроблені моделі дають нову інформацію щодо фізики взаємодії сонячного вітру та магнітосфери Землі. 